\title{
Characterizations of Generalized Exponential Trichotomies for Linear Discrete-time Systems
}

\author{
Ioan-Lucian Popa, Traian Ceauşu, \\ Ovidiu Bagdasar and Ravi P. Agarwal
}

\begin{abstract}
The concept of generalized exponential trichotomy for linear timevarying systems is investigated in relationship with the classical notion of uniform exponential trichotomy. Some key properties of generalized exponential trichotomy are explored through supplementary projections. These results are also extended to the case of projection sequences, while certain applications for adjoint systems are suggested.
\end{abstract}

\section{Introduction}

The trichotomy concept involves splitting the state space at any moment into three subspaces (a stable, unstable and a central one) and represents the most complex description of the asymptotic behavior of linear time-varying (LTV) systems. The first notable study on the uniform exponential trichotomy (UET) for discrete-time LTV systems was done by S. Elaydi and K. Janglajew in [5]. Numerous extensions have followed. For example in [7], [12], [13] (and the references therein), various nonuniform exponential concepts are presented, where some exponential loss of hyperbolicity along the trajectories is allowed. Some nonuniform concepts of polynomial type are presented in [15].

Motivated by results obtained by A. Castaneda and G. Robledo in [2] for generalized exponential dichotomy (GED) for difference equations, this notion

Key Words: difference equations, (generalized) exponential trichotomy, discrete-time linear time-varying systems.

2010 Mathematics Subject Classification: Primary 34D09, 34D05; Secondary 39A06, $93 \mathrm{C} 55$.

Received: 26.06.2018

Accepted: 30.07 .2018 
was extended to generalized exponential trichotomy (GET) in [10], where we have established the relation between this notion and the classical (uniform) exponential trichotomy from [5]. It should be noted that our notion of GET is not a kind of nonuniform hyperbolicity. In fact our notion represents a kind of uniform hyperbolicity. In this context, we can point out some important results obtained in this direction in [2], [6].

In this paper we give a simple and concrete example illustrating the relationship between the concepts of UET and GET. Also, motivated by the lead given in [5], we present some theorems of characterization for discrete-time LTV systems in terms of GET. More precisely, we will show in Section 2 how the mutual orthogonality property matrix projections can be replaced for the case of the GET property. Also, these characterizations are extended in Section 3 for the case of invariant projection sequences. Subsequently, in the last section of the paper a necessary and sufficient condition for GET property for the dual system is developed. This paper is a companion of our earlier work [11] where some preliminary results have been presented.

Notations. The notations used in this paper are generally standard. For the readers' convenience we recall some of them: $\mathbb{Z}$ denotes the set of real integers, $\mathbb{Z}_{+}$is the set of all $n \in \mathbb{Z}, n \geq 0, \mathbb{Z}_{-}$is the set of all $n \in \mathbb{Z}, n \leq 0$, while $\mathbb{R}$ denotes the set of real numbers and $\|$.$\| represents a matrix norm.$

\section{Generalized exponential trichotomy}

Let us consider the LTV system

$$
x_{n+1}=A_{n} x_{n}, \quad n \in \mathbb{Z},
$$

where $\left(A_{n}\right)_{n \in \mathbb{Z}}$ is a sequence of $d \times d$ invertible matrices. By $W_{n}$ we denote the fundamental matrix of $(\mathfrak{A})$, i.e., $W_{n+1}=A_{n} W_{n}$ and $W_{0}=I$, where $I$ represents the identity matrix. Further on, we shall consider a strictly positive sequence $\left(a_{n}\right)_{n \in \mathbb{Z}}$ satisfying the properties

$$
\begin{aligned}
& \sum_{j=p}^{q} a_{j} \rightarrow+\infty \text { as } q \rightarrow+\infty \text { for fixed } p \in \mathbb{Z}, \\
& \sum_{j=p}^{q} a_{j} \rightarrow+\infty \text { as } p \rightarrow-\infty \text { for fixed } q \in \mathbb{Z} .
\end{aligned}
$$

Definition 2.1. ([10]) The LTV system ( $\mathfrak{A})$ admits a generalized exponential trichotomy $(\mathrm{GET})$ on $\mathbb{Z}$ if there exist projections $\left(P^{i}\right), i \in\{1,2,3\}$, satisfying

$$
P^{1}+P^{2}+P^{3}=I \text { and } P^{i} P^{j}=P^{j} P^{i}=0,
$$


for all $i, j \in\{1,2,3\}$ with $i \neq j$, together with the constants $K \geq 1, p \in(0,1)$, and a strictly positive sequence $\left(a_{n}\right)_{n \in \mathbb{Z}}$ satisfying (1) and (2) such that

$$
\begin{gathered}
\left\|W_{n} P^{1} W_{m}^{-1}\right\| \leq K p^{\sum_{j=m}^{n} a_{j}}, \quad n \geq m, \\
\left\|W_{n} P^{2} W_{m}^{-1}\right\| \leq K p^{\sum_{j=n}^{m} a_{j}}, \quad m \geq n, \\
\left\|W_{n} P^{3} W_{m}^{-1}\right\| \leq K p^{\sum_{j=n}^{m} a_{j}}, \quad 0 \geq m \geq n, \\
\left\|W_{n} P^{3} W_{m}^{-1}\right\| \leq K p^{\sum_{j=m}^{n} a_{j}}, \quad n \geq m \geq 0 .
\end{gathered}
$$

In order to simplify the notations further we will denote by $t_{m n}=\sum_{k=m}^{n} a_{k}$, for all $m, n \in \mathbb{Z}$, with $n \geq m$. Also, we point out that the projections $P^{i}$, $i \in\{1,2,3\}$, satisfying relation (3) are called supplementary.

One may notice that the LTV system $(\mathfrak{A})$ admits a generalized exponential dichotomy (GED) if it admits a GET with $P^{3}=0$, for all $n \in \mathbb{Z}$. The notion of GED has been introduced by A. Castaneda and G. Robledo in [2]. For the particular case when $a_{j}=\alpha>0$, for any $j \in \mathbb{Z}$, we obtain the notion of $\alpha$-exponential dichotomy from [8]. For a deeper discussion about discrete dichotomies we refer the reader to [1], [2], [4], [9] and the references therein.

There are examples of GETs for which $a_{j}$ cannot be replaced by a constant $\alpha$, as shown by the example below. For more details one may consult [10]. Consider a sequence $\left(b_{n}\right)_{n \in \mathbb{Z}}$ satisfying the following properties

- If $n \in \mathbb{N}$ then $0<b_{0} \leq b_{1} \leq \ldots \leq b_{n} \leq b_{n+1} \leq \ldots<1$ and $\left(b_{n}\right)_{n \in \mathbb{Z}}$ monotonically increasing to 1 as $n \rightarrow \infty$.

- If $n \in \mathbb{Z} \backslash \mathbb{N}$ then $b_{n}=b_{-n}$.

We consider $\left(c_{n}\right)_{n \in \mathbb{Z}}$ defined by $c_{n}= \begin{cases}b_{n} & n \in \mathbb{Z} \\ 1 / b_{n} & n \in \mathbb{Z} \backslash \mathbb{N} .\end{cases}$

It is shown in particular that on $\mathbb{R}^{3}$ endowed with the Euclidean norm that the LTV system generated by

$$
A_{n}=\left(\begin{array}{ccc}
b_{n} & 0 & 0 \\
0 & 1 / b_{n} & 0 \\
0 & 0 & c_{n}
\end{array}\right),
$$

has a GET and not an $\alpha$-exponential one. 
Proposition 2.1. The LTV system (A) has a GET if and only if there exist two projections $T^{1}$ and $T^{2}$ satisfying $T^{1} T^{2}=T^{2} T^{1}$ and $T^{1}+T^{2}-T^{1} T^{2}=$ $I=T^{1}+T^{2}-T^{2} T^{1}$, some constants $D \geq 1, p \in(0,1)$ and a strictly positive sequence $\left(a_{n}\right)_{n \in \mathbb{Z}}$ satisfying (1) and (2), such that

$$
\begin{gathered}
\left\|W_{n}\left(I-T^{2}\right) W_{m}^{-1}\right\| \leq D p^{t_{m n}}, \quad n \geq m, \\
\left\|W_{n}\left(I-T^{1}\right) W_{m}^{-1}\right\| \leq D p^{t_{n m}}, \quad m \geq n, \\
\left\|W_{n} T^{2} W_{m}^{-1}\right\| \leq D p^{t_{n m}}, \quad 0 \geq m \geq n, \\
\left\|W_{n} T^{1} W_{m}^{-1}\right\| \leq D p^{t_{m n}}, \quad n \geq m \geq 0,
\end{gathered}
$$

Proof. Necessity. We consider $T^{1}=P^{1}+P^{3}$ and $T^{2}=P^{2}+P^{3}$. It can be easily seen that $T^{1}$ and $T^{2}$ are projections and $T^{1} T^{2}=T^{2} T^{1}=P^{3}$. From $P^{1}+P^{2}+P^{3}=I$ we have that

$$
T^{1}+T^{2}-T^{1} T^{2}=I=T^{1}+T^{2}-T^{2} T^{1} .
$$

Also, we have that

$$
I-T^{2}=T^{1}-T^{1} T^{2}=P^{1} \text { and } I-T^{1}=T^{2}-T^{2} T^{1}=P^{2} .
$$

By direct calculation from equation (4) we obtain (8) and by (5) we get (9). Finally, by equations (5) and (6) one obtains (10), and similarly by (4) and (7) it follows that (11) holds.

Sufficiency. Let $P^{1}=I-T^{2}, P^{2}=I-T^{1}$ and $P^{3}=T^{2} T^{1}=T^{1} T^{2}$. Then $P^{i}, i \in\{1,2,3\}$ are supplementary projections.

First note that (8) implies (4) and (9) implies (5). Now, from (9) for $m=n$ we obtain that

$$
\begin{aligned}
\left\|W_{n} T^{1} W_{n}^{-1}\right\| & =\left\|W_{n}\left(-T^{1}\right) W_{n}^{-1}\right\| \leq\left\|W_{n}\left(I-T^{1}\right) W_{n}^{-1}\right\|+\|I\| \\
& \leq D p^{a_{n}}+1 \leq 2 D .
\end{aligned}
$$

Analogously, from (8) for $m=n$ we get

$$
\begin{aligned}
\left\|W_{n} T^{2} W_{n}^{-1}\right\| & =\left\|W_{n}\left(-T^{2}\right) W_{n}^{-1}\right\| \leq\left\|W_{n}\left(I-T^{2}\right) W_{n}^{-1}\right\|+\|I\| \\
& \leq D p^{a_{n}}+1 \leq 2 D .
\end{aligned}
$$

Further, for $0 \geq m \geq n$, by (10) and the two previous inequalities we obtain

$$
\begin{aligned}
\left\|W_{n} P^{3} W_{m}^{-1}\right\| & =\left\|W_{n} T^{1} T^{2} W_{m}^{-1}\right\|=\left\|W_{n} T^{1} W_{n}^{-1} W_{n} T^{2} W_{m}^{-1}\right\| \\
& \leq 2 D^{2} p^{t_{n m}} .
\end{aligned}
$$

Finally, for $n \geq m \geq 0$ taking into account (11) we have that

$$
\left\|W_{n} P^{3} W_{m}^{-1}\right\| \leq 2 D^{2} p^{t_{m n}} .
$$

which ends the proof. 
Proposition 2.2. The LTV system (A) has a GET if and only if there exist two projections $G^{1}$ and $G^{2}$ satisfying $G^{1} G^{2}=G^{2} G^{1}=G^{1}$, some constants $D \geq 1, p \in(0,1)$ and a strictly positive sequence $\left(a_{n}\right)_{n \in \mathbb{Z}}$ satisfying (1) and (2), such that

$$
\begin{gathered}
\left\|W_{n} G^{1} W_{m}^{-1}\right\| \leq D p^{t_{m n}}, \quad n \geq m, \\
\left\|W_{n}\left(I-G^{2}\right) W_{m}^{-1}\right\| \leq D p^{t_{n m}}, \quad m \geq n, \\
\left\|W_{n}\left(I-G^{1}\right) W_{m}^{-1}\right\| \leq D p^{t_{n m}}, \quad 0 \geq m \geq n, \\
\left\|W_{n} G^{2} W_{m}^{-1}\right\| \leq D p^{t_{m n}}, \quad n \geq m \geq 0 .
\end{gathered}
$$

Proof. Let $T^{1}$ and $T^{2}$ be the projections considered in Proposition 2.1. Define the projections $G^{1}$ and $G^{2}$ by $G^{1}=I-T^{2}$ and $G^{2}=T^{1}$. Clearly, these projections satisfy $G^{1} G^{2}=G^{2} G^{1}=G^{1}$.

Conversely, suppose that $G^{1}$ and $G^{2}$ are two projections satisfying the condition $G^{1} G^{2}=G^{2} G^{1}=G^{1}$. By letting $T^{1}=G^{2}$ and $T^{2}=I-G^{1}$, it follows that $T^{1} T^{2}=T^{2} T^{1}=G^{2}-G^{1}$ is a projection because

$$
\left(T^{1} T^{2}\right)^{2}=\left(G^{2}-G^{1}\right)^{2}=G^{2}-G^{1}=T^{1} T^{2} .
$$

Moreover,

$$
T^{1}+T^{2}-T^{1} T^{2}=T^{1}+T^{2}-T^{2} T^{1}=I .
$$

Therefore, the equivalence between the equations (8)-(11) and (12)-(15) can be directly obtained.

Proposition 2.3. For every $m \in \mathbb{Z}^{*}$ we have that

$$
W_{m}= \begin{cases}A_{m-1} A_{m-2} \cdots A_{1} A_{0}, & \text { if } m>0 \\ \left(A_{-1} A_{-2} \cdots A_{m}\right)^{-1}, & \text { if } m<0 .\end{cases}
$$

Proof. If $m>0$, then

$$
\begin{aligned}
W_{m} & =A_{m-1} W_{m-1}=A_{m-1} A_{m-2} W_{m-1}=\ldots=A_{m-1} A_{m-2} \cdots A_{1} A_{0} W_{0} \\
& =A_{m-1} A_{m-2} \cdots A_{1} A_{0}
\end{aligned}
$$

while for $m<0$ we have that

$$
\begin{aligned}
W_{m} & =A_{m}^{-1} W_{m+1}=A_{m}^{-1} A_{m+1}^{-1} W_{m+2}=\ldots=A_{m}^{-1} A_{m+1}^{-1} \cdots A_{-1}^{-1} W_{0} \\
& =A_{m}^{-1} A_{m+1}^{-1} \cdots A_{-1}^{-1}=\left(A_{-1} A_{-2} \cdots A_{m}\right)^{-1}
\end{aligned}
$$




\section{Generalized exponential trichotomy with projection sequences}

The principal aim of this section is to give a characterization of GET in terms of two invariant projection sequences. We begin with some definitions.

A sequence $\left(P_{n}\right)_{n \in \mathbb{Z}}$ is called a projection sequence if $\left(P_{n}\right)^{2}=P_{n}$, for $n \in \mathbb{Z}$. A projection sequence $\left(P_{n}\right)_{n \in \mathbb{Z}}$ with the property $P_{n+1} A_{n}=A_{n} P_{n}$, for all $n \in \mathbb{Z}$ is called invariant for the LTV system $(\mathfrak{A})$. Three projection sequences $\left(P_{n}^{i}\right)_{n \in \mathbb{Z}}, i \in\{1,2,3\}$, are called supplementary if

$$
\begin{gathered}
P_{n}^{1}+P_{n}^{2}+P_{n}^{3}=I, \text { for } n \in \mathbb{Z}, \\
P_{n}^{i} P_{n}^{j}=0, \text { for } n \in \mathbb{Z} \text { and } i, j \in\{1,2,3\}, i \neq j .
\end{gathered}
$$

Proposition 3.1. Let $P$ be a projection. If $S_{m}=W_{m} P W_{m}^{-1}$, for every $m \in \mathbb{Z}$, then $\left(S_{m}\right)_{m \in \mathbb{Z}}$ is a projection sequence such that

$$
S_{m+1} A_{m}=A_{m} S_{m},
$$

for all $m \in \mathbb{Z}$.

Proof. Let $m \in \mathbb{Z}$. It is easily seen that $S_{m}^{2}=S_{m}$ and

$$
\begin{aligned}
S_{m+1} A_{m} & =W_{m+1} P W_{m+1}^{-1} A_{m}=\left(A_{m} W_{m}\right) P\left(W_{m}^{-1} A_{m}^{-1}\right) A_{m} \\
& =A_{m}\left(W_{m} P W_{m}^{-1}\right)\left(A_{m}^{-1} A_{m}\right)=A_{m} S_{m} .
\end{aligned}
$$

A property of the invariant projection sequences was reported in [14].

Proposition 3.2. If $\left(P_{n}\right)_{n \in \mathbb{Z}}$ is an invariant projection sequence for the LTV system (A), then $P_{m}=W_{m} P_{0} W_{m}^{-1}$ for every $m \in \mathbb{Z}$.

Proof. If $m>0$, then

$$
\begin{aligned}
P_{m} & =A_{m-1} P_{m-1} A_{m-1}^{-1}=A_{m-1} A_{m-2} P_{m-2} A_{m-2}^{-1} A_{m-1}^{-1} \\
& =\ldots=A_{m-1} A_{m-2} \cdots A_{1} A_{0} P_{0} A_{0}^{-1} A_{1}^{-1} \cdots A_{m-2}^{-1} A_{m-1}^{-1} \\
& =W_{m} P_{0} W_{m}^{-1}
\end{aligned}
$$

while for $m<0$ we have

$$
\begin{aligned}
P_{m} & =A_{m}^{-1} P_{m+1} A_{m}=A_{m}^{-1} A_{m+1}^{-1} P_{m+2} A_{m+1} A_{m} \\
& =\ldots=A_{m}^{-1} A_{m+1}^{-1} A_{m+2}^{-1} \cdots A_{-1}^{-1} P_{0} A_{-1} A_{-2} \cdots A_{m+2} A_{m+1} A_{m} \\
& =W_{m} P_{0} W_{m}^{-1} .
\end{aligned}
$$


The following theorems are the main results of the paper.

Theorem 3.1. The LTV system (A) has a GET if and only if there exist invariant and supplementary projection sequences $\left(S_{m}^{k}\right)_{m \in \mathbb{Z}}, k \in\{1,2,3\}$, some constants $D \geq 1, p \in(0,1)$ and a sequence $\left(a_{n}\right)_{n \in \mathbb{Z}}$ satisfying (1) and (2), such that

$$
\begin{gathered}
\left\|W_{n} W_{m}^{-1} S_{m}^{1}\right\| \leq D p^{t_{m n}}, \quad n \geq m, \\
\left\|W_{n} W_{m}^{-1} S_{m}^{2}\right\| \leq D p^{t_{n m}}, \quad m \geq n, \\
\left\|W_{n} W_{m}^{-1} S_{m}^{3}\right\| \leq D p^{t_{n m}}, \quad 0 \geq m \geq n, \\
\left\|W_{n} W_{m}^{-1} S_{m}^{3}\right\| \leq D p^{t_{m n}}, \quad n \geq m \geq 0 .
\end{gathered}
$$

Proof. Necessity. Let $P^{k}, k \in\{1,2,3\}$ be projections given in Definition 2.1. For every $m \in \mathbb{Z}$ we consider $S_{m}^{k}=W_{m} P^{k} W_{m}^{-1}, k \in\{1,2,3\}$. According to Proposition 3.1, $\left(S_{m}^{k}\right)_{m \in \mathbb{Z}}$ are projection sequences which satisfy the invariant property $S_{m+1}^{k} A_{m}=A_{m} S_{m}^{k}, m \in \mathbb{Z}$. For all $m \in \mathbb{Z}$ and $i, j \in\{1,2,3\}$ with $i \neq j$, one has

$$
\begin{gathered}
S_{m}^{1}+S_{m}^{2}+S_{m}^{3}=W_{m}\left(P^{1}+P^{2}+P^{3}\right) W_{m}^{-1}=I, \\
S_{m}^{j} S_{m}^{i}=W_{m} P^{j} W_{m}^{-1} W_{m} P^{i} W_{m}^{-1}=0, \\
S_{m}^{i} S_{m}^{j}=W_{m} P^{i} W_{m}^{-1} W_{m} P^{j} W_{m}^{-1}=0,
\end{gathered}
$$

hence the projections are also supplementary.

Let $m, n \in \mathbb{Z}$. Taking into account that $P^{k} W_{m}^{-1}=W_{m}^{-1} S_{m}^{k}$ we obtain that

$$
W_{n} P^{k} W_{m}^{-1}=W_{n} W_{m}^{-1} S_{m}^{k},
$$

for all $k \in\{1,2,3\}$. Finally, (18)-(21) follow immediately by (4)-(7).

Sufficiency. Let $k \in\{1,2,3\}$. Based on Proposition 3.2 we have that $S_{m}^{k}=$ $W_{m} S_{0}^{k} W_{m}^{-1}$, or equivalently, $W_{m}^{-1} S_{m}^{k}=S_{0}^{k} W_{m}^{-1}$. This leads to $W_{n} W_{m}^{-1} S_{m}^{k}=$ $W_{n} S_{0}^{k} W_{m}^{-1}$, for all $n, m \in \mathbb{Z}$. Setting $P^{k}=S_{0}^{k}$ we have that projections $P^{k}$, $k \in\{1,2,3\}$, verifies the conditions from Definition 2.1. Thus, we obtain the equivalence between the equations (18)-(21) and (4)-(7).

Remark. (a) By Definition 2.1, for $m=n=0$ we have $\left\|P^{k}\right\| \leq K p^{a_{0}} \leq K$, for all $k \in\{1,2,3\}$.

(b) By Theorem 3.1, for $m=n$ we have $\left\|S_{m}^{k}\right\| \leq D p^{a_{m}} \leq D$, for all $k \in$ $\{1,2,3\}$. 
Theorem 3.2. The LTV system ( $\mathfrak{A})$ has a GET if and only if there exist two invariant projection sequences $\left(Q_{n}^{i}\right)_{n \in \mathbb{Z}}, i \in\{1,2\}$, some constants $K \geq 1$, $p \in(0,1)$ and a sequence $\left(a_{n}\right)_{n \in \mathbb{Z}}$ satisfying (1) and (2), such that

$$
\begin{gathered}
Q_{n}^{1}+Q_{n}^{2}-Q_{n}^{1} Q_{n}^{2}=I, \quad Q_{n}^{1} Q_{n}^{2}=Q_{n}^{2} Q_{n}^{1}, \text { for all } n \in \mathbb{Z}, \\
\left\|W_{n} W_{m}^{-1} Q_{m}^{1}\right\| \leq M p^{t_{m n}}, \quad n \geq m \geq 0 \\
\left\|W_{n} W_{m}^{-1} Q_{m}^{2}\right\| \leq M p^{t_{n m}}, \quad 0 \geq m \geq n \\
\left\|W_{n} W_{m}^{-1}\left(I-Q_{m}^{1}\right)\right\| \leq M p^{t_{n m}}, \quad m \geq n \\
\left\|W_{n} W_{m}^{-1}\left(I-Q_{m}^{2}\right)\right\| \leq M p^{t_{m n}}, \quad n \geq m .
\end{gathered}
$$

Proof. Necessity. Let $n \in \mathbb{Z}$. We consider $Q_{n}^{1}=S_{n}^{1}+S_{n}^{3}$ and $Q_{n}^{2}=S_{n}^{2}+S_{n}^{3}$. One can easily see that $Q_{n}^{1} Q_{n}^{2}=Q_{n}^{2} Q_{n}^{1}=S_{n}^{3}$ and $Q_{n}^{1}$ and $Q_{n}^{2}$ are invariant projection sequences for the LTV system $(\mathfrak{A})$. Also, using the supplementary property we have that

$$
Q_{n}^{1}+Q_{n}^{2}-Q_{n}^{1} Q_{n}^{2}=Q_{n}^{1}+Q_{n}^{2}-Q_{n}^{2} Q_{n}^{1}=I
$$

Further, one can easily observe that $I-Q_{n}^{1}=S_{n}^{2}$ and $I-Q_{n}^{2}=S_{n}^{1}$, hence $\left\|Q_{n}^{1}\right\| \leq\left\|S_{n}^{1}\right\|+\left\|S_{n}^{3}\right\| \leq 2 D$, respectively $\left\|Q_{n}^{2}\right\| \leq\left\|S_{n}^{2}\right\|+\left\|S_{n}^{3}\right\| \leq 2 D$.

We have to consider the following cases.

(1) For $n \geq m \geq 0$, using (18) and (21) we have that

$$
\left\|W_{n} W_{m}^{-1} Q_{m}^{1}\right\|=\left\|W_{n} W_{m}^{-1}\left(S_{m}^{1}+S_{m}^{3}\right)\right\| \leq 2 D p^{t_{m n}} .
$$

(2) For $0 \geq m \geq n$ using (19) and (20) we get

$$
\left\|W_{n} W_{m}^{-1} Q_{m}^{2}\right\|=\left\|W_{n} W_{m}^{-1}\left(S_{m}^{2}+S_{m}^{3}\right)\right\| \leq 2 D p^{t_{n m}} .
$$

(3) If $m \geq n$, then using (19) we obtain

$$
\left\|W_{n} W_{m}^{-1}\left(I-Q_{m}^{1}\right)\right\|=\left\|W_{n} W_{m}^{-1} S_{m}^{2}\right\| \leq D p^{t_{n m}} .
$$

(4) Finally, for $n \geq m$ using (18) we deduce that

$$
\left\|W_{n} W_{m}^{-1}\left(I-Q_{m}^{2}\right)\right\|=\left\|W_{n} W_{m}^{-1} S_{m}^{1}\right\| \leq D p^{t_{m n}} .
$$

Sufficiency. For each $n \in \mathbb{Z}$ we consider $S_{n}^{1}=I-Q_{n}^{2}, S_{n}^{2}=I-Q_{n}^{1}$ and $S_{n}^{3}=Q_{n}^{1} Q_{n}^{2}=Q_{n}^{2} Q_{n}^{1}$. We firstly observe that

$$
S_{n}^{1}+S_{n}^{2}+S_{n}^{3}=I-Q_{n}^{2}+I-Q_{n}^{1}+Q_{n}^{1} Q_{n}^{2}=I-Q_{n}^{1}-Q_{n}^{2}+Q_{n}^{1} Q_{n}^{2}+I=I,
$$


and $S_{n}^{i} S_{n}^{j}=S_{n}^{j} S_{n}^{i}=0$, for all $i, j \in\{1,2,3\}$ with $i \neq j$. Next, we have that

$$
S_{n+1}^{1} A_{n}=\left(I-Q_{n+1}^{2}\right) A_{n}=A_{n}-Q_{n+1}^{2} A_{n}=A_{n}\left(I-Q_{n}^{2}\right)=A_{n} S_{n}^{1} .
$$

Similarly, we have that $S_{n+1}^{i} A_{n}=A_{n} S_{n}^{i}$, with $i \in\{2,3\}$. Therefore, $S_{n}^{i}$, $i \in\{1,2,3\}$ are invariant projection sequences for the system $(\mathfrak{A})$. Also observe that for $m=n$ condition (25) implies that $\left\|I-Q_{m}^{1}\right\| \leq M p^{a_{m}} \leq M$, i.e., $\left\|Q_{m}^{1}\right\| \leq 1+M \leq 2 M$. Similarly, from (26) we have that $\left\|Q_{m}^{2}\right\| \leq 1+M \leq 2 M$.

In order to establish equivalence, first note that (26) and (25) clearly imply (18) and (19). On the other hand, setting $0 \geq m \geq n$, (24) becomes

$$
\left\|W_{n} W_{m}^{-1} S_{m}^{3}\right\| \leq\left\|W_{n} W_{m}^{-1} Q_{m}^{2}\right\| \cdot\left\|Q_{m}^{1}\right\| \leq 2 M^{2} p^{t_{n m}} .
$$

Similarly, setting $n \geq m \geq 0$, (23) becomes

$$
\left\|W_{n} W_{m}^{-1} S_{m}^{3}\right\| \leq\left\|W_{n} W_{m}^{-1} Q_{m}^{1}\right\| \cdot\left\|Q_{m}^{2}\right\| \leq 2 M^{2} p^{t_{m n}} .
$$

Finally, applying Theorem 3.1 we obtain that system (A) admits a GET, which ends the proof.

Theorem 3.3. The LTV system (A) has a GET if and only if there exist two invariant projection sequences $\left(R_{n}^{i}\right)_{n \in \mathbb{Z}}, i \in\{1,2\}$, some constants $K \geq 1$, $p \in(0,1)$ and a sequence $\left(a_{n}\right)_{n \in \mathbb{Z}}$ satisfying (1) and (2), such that

$$
\begin{gathered}
R_{n}^{1} R_{n}^{2}=R_{n}^{2} R_{n}^{1}=R_{n}^{2}, \text { for all } n \in \mathbb{Z}, \\
\left\|W_{n} W_{m}^{-1} R_{m}^{1}\right\| \leq K p^{t_{m n}}, \quad n \geq m \geq 0, \\
\left\|W_{n} W_{m}^{-1} R_{m}^{2}\right\| \leq K p^{t_{m n}}, \quad 0 \geq m \geq n, \\
\left\|W_{n} W_{m}^{-1}\left(I-R_{m}^{1}\right)\right\| \leq K p^{t_{n m}}, \quad m \geq n, \\
\left\|W_{n} W_{m}^{-1}\left(I-R_{m}^{2}\right)\right\| \leq K p^{t_{m n}}, \quad n \geq m .
\end{gathered}
$$

Proof. Necessity. Let $n \in \mathbb{Z}$. We set $R_{n}^{1}=Q_{n}^{1}$ and $R_{n}^{2}=I-Q_{n}^{2}$. If follows from (22), that

$$
R_{n}^{1} R_{n}^{2}=Q_{n}^{1}-Q_{n}^{1} Q_{n}^{2}=I-Q_{n}^{2}=R_{n}^{2},
$$

and

$$
R_{n}^{2} R_{n}^{1}=Q_{n}^{1}-Q_{n}^{2} Q_{n}^{1}=I-Q_{n}^{2}=R_{n}^{2} .
$$

It can easily be checked the invariant property for $R_{n}^{i}, i \in\{1,2\}$. Also, we have that $\left\|R_{n}^{1}\right\| \leq M$ and $\left\|R_{n}^{2}\right\| \leq 1+M$. Therefore, the equivalence between the equations (23)-(26) and (28)-(31) can be directly obtained. that

Sufficiency. Let $n \in \mathbb{Z}$. Setting $Q_{n}^{1}=R_{n}^{1}$ and $Q_{n}^{2}=I-R_{n}^{2}$ one can show

$$
Q_{n}^{1} Q_{n}^{2}=R_{n}^{1}-R_{n}^{1} R_{n}^{2}=R_{n}^{1}-R_{n}^{2},
$$


and

$$
Q_{n}^{2} Q_{n}^{1}=R_{n}^{1}-R_{n}^{2} R_{n}^{1}=R_{n}^{1}-R_{n}^{2} .
$$

Furthermore, $Q_{n}^{1} Q_{n}^{2}$ is a projection sequence satisfying $Q_{n}^{1}+Q_{n}^{2}-Q_{n}^{1} Q_{n}^{2}=I$. Hence, $Q_{n}^{1}, Q_{n}^{2}$ and $Q_{n}^{1} Q_{n}^{2}$ are projection sequences satisfying (22). We also note that for $m=n$, by (29) we have that $\left\|R_{n}^{2}\right\| \leq K p^{a_{m}} \leq K$, while from (30) we obtain $\left\|R_{n}^{1}\right\| \leq 2 K$.

Finally, (28)-(31) implies (23)-(26), which completes the proof.

\section{Generalized exponential trichotomy for adjoint system}

If $\left(A_{n}\right)_{n \in \mathbb{Z}}$ is a sequence of $d \times d$ invertible matrices with complex elements, then the adjoint system associated to $(\mathfrak{A})$ is given by

$$
y_{n}=A_{n}^{*} y_{n+1}, \quad n \in \mathbb{Z}
$$

or, in equivalent form

$$
y_{n+1}=\left(A_{n}^{*}\right)^{-1} y_{n}, \quad n \in \mathbb{Z} .
$$

If $V_{n}$ is the fundamental matrix of $(\mathfrak{Y})$, then $V_{n+1}=\left(A_{n}^{*}\right)^{-1} V_{n}$, and $V_{0}=I$. Inductively, for $m>0$ we have

$$
\begin{aligned}
V_{m} & =\left(A_{m-1}^{*}\right)^{-1}\left(A_{m-2}^{*}\right)^{-1} \cdots\left(A_{1}^{*}\right)^{-1}\left(A_{0}^{*}\right)^{-1}=\left(A_{0}^{*} A_{1}^{*} \cdots A_{m-1}^{*}\right)^{-1} \\
& =\left(\left(A_{m-1} A_{m-2} \cdots A_{1} A_{0}\right)^{*}\right)^{-1}=\left(W_{m}^{*}\right)^{-1} .
\end{aligned}
$$

In the same way, for $m<0$ we have that $V_{m}=\left(W_{m}^{*}\right)^{-1}$.

Proposition 4.1. Let $P$ be a projection. For every $m, n \in \mathbb{Z}$ we have

$$
\left\|W_{n} P W_{m}^{-1}\right\|=\left\|V_{m} P^{*} V_{n}^{-1}\right\| .
$$

Proof. We have that

$$
\left\|W_{n} P W_{m}^{-1}\right\|=\left\|\left(W_{n} P W_{m}^{-1}\right)^{*}\right\|=\left\|\left(W_{m}^{-1}\right)^{*} P^{*} W_{n}^{*}\right\|=\left\|V_{m} P^{*} V_{n}^{-1}\right\| .
$$

Remark. In what follows, we will describe characterizations of GET property for the dual system $(\mathfrak{Y})$ with adjoint projections $\left(P^{j}\right)^{*}, j \in\{1,2,3\}$. The arguments of the proof are similar to the arguments used for Proposition 2.1 and thus omitted. 
Proposition 4.2. Let (A) be a system admitting a GET with constants $K \geq 1$, $p \in(0,1)$, strictly positive sequence $\left(a_{n}\right)_{n \in \mathbb{Z}}$, and projections $P^{k}, k \in\{1,2,3\}$ considered in Definition 2.1. Then the adjoint system (Y) also has a GET with the same constants and projections $\left(P^{j}\right)^{*}, j \in\{1,2,3\}$. More precisely, we have

$$
\begin{gathered}
\left\|V_{m}\left(P^{1}\right)^{*} V_{n}^{-1}\right\| \leq K p^{t_{m n}}, \quad n \geq m, \\
\left\|V_{m}\left(P^{2}\right)^{*} V_{n}^{-1}\right\| \leq K p^{t_{n m}}, \quad m \geq n, \\
\left\|V_{m}\left(P^{3}\right)^{*} V_{n}^{-1}\right\| \leq K p^{t_{n m}}, \quad 0 \geq m \geq n, \\
\left\|V_{m}\left(P^{3}\right)^{*} V_{n}^{-1}\right\| \leq K p^{t_{m n}}, \quad n \geq m \geq 0 .
\end{gathered}
$$

Proposition 4.3. Let (A) be a system admitting a GET with constants $D \geq 1$, $p \in(0,1)$, strictly positive sequence $\left(a_{n}\right)_{n \in \mathbb{Z}}$, and projections $T^{k}, k \in\{1,2\}$ considered in Proposition 2.1. Then the adjoint system (Y) also has a GET with the same constants and projections $\left(\left(T^{j}\right)^{*}\right), j \in\{1,2\}$. More precisely, we have

$$
\begin{gathered}
\left\|V_{m}\left(I-\left(T^{2}\right)^{*}\right) V_{n}^{-1}\right\| \leq D p^{t_{m n}}, \quad n \geq m \\
\left\|V_{m}\left(I-\left(T^{1}\right)^{*}\right) V_{n}^{-1}\right\| \leq D p^{t_{n m}}, \quad m \geq n \\
\left\|V_{m}\left(T^{2}\right)^{*} V_{n}^{-1}\right\| \leq D p^{t_{n m}}, \quad 0 \geq m \geq n, \\
\left\|V_{m}\left(T^{1}\right)^{*} V_{n}^{-1}\right\| \leq D p^{t_{m n}}, \quad n \geq m \geq 0 .
\end{gathered}
$$

Let $m, n \in \mathbb{Z}$, with $m \geq n$ and consider

$$
A_{m}^{n}= \begin{cases}A_{m-1} A_{m-2} \cdots A_{n}, & \text { if } m>n, \\ I, & \text { if } m=n .\end{cases}
$$

For $m, n \in \mathbb{Z}$ one obtains

$$
W_{n} W_{m}^{-1}= \begin{cases}A_{n}^{m}, & \text { if } n \geq m, \\ \left(A_{m}^{n}\right)^{-1}, & \text { if } m \geq n .\end{cases}
$$

Proposition 4.4. If $\left(P_{n}\right)_{n \in \mathbb{Z}}$ is an invariant projection sequence for the $L T V$ system ( $\mathfrak{A})$, then for every $n \geq m$ we have

$$
A_{n}^{m} P_{m}=P_{n} A_{n}^{m},
$$

which is equivalent to

$$
P_{m}\left(A_{n}^{m}\right)^{-1}=\left(A_{n}^{m}\right)^{-1} P_{n} .
$$

Proposition 4.5. If $\left(P_{n}\right)_{n \in \mathbb{Z}}$ is a invariant projection sequences for the $L T V$ system ( $\mathfrak{A})$ then for every $n, m \in \mathbb{Z}$ we have that

$$
\left\|W_{n} W_{m}^{-1} P_{m}\right\|=\left\|V_{m} V_{n}^{-1} P_{n}^{*}\right\| .
$$


Proof. If $n \geq m$, then

$$
\begin{aligned}
\left\|W_{n} W_{m}^{-1} P_{m}\right\| & =\left\|\left(W_{n} W_{m}^{-1} P_{m}\right)^{*}\right\|=\left\|\left(A_{n}^{m}\right)^{*} P_{n}^{*}\right\| \\
& =\left\|\left(W_{n} W_{m}^{-1}\right)^{*} P_{n}^{*}\right\|=\left\|\left(W_{m}^{-1}\right)^{*} W_{n}^{*} P_{n}^{*}\right\|=\left\|V_{m} V_{n}^{-1} P_{n}^{*}\right\|,
\end{aligned}
$$

and if $m \geq n$, then we have

$$
\begin{aligned}
\left\|W_{n} W_{m}^{-1} P_{m}\right\| & =\left\|\left(W_{n} W_{m}^{-1} P_{m}\right)^{*}\right\|=\left\|\left(\left(A_{m}^{n}\right)^{-1}\right)^{*} P_{n}^{*}\right\| \\
& =\left\|\left(W_{n} W_{m}^{-1}\right)^{*} P_{n}^{*}\right\|=\left\|\left(W_{m}^{-1}\right)^{*} W_{n}^{*} P_{n}^{*}\right\|=\left\|V_{m} V_{n}^{-1} P_{n}^{*}\right\| .
\end{aligned}
$$

Proposition 4.6. If $\left(P_{n}\right)_{n \in \mathbb{Z}}$ is an invariant projection sequence for the $L T V$ system ( $\mathfrak{A})$, then $\left(P_{n}^{*}\right)_{n \in \mathbb{Z}}$ is an invariant projection sequence for the LTV system (Y)).

Proof. Let $n \in \mathbb{Z}$. Using the invariant property $P_{n+1} A_{n}=A_{n} P_{n}$ we have that $A_{n}^{*} P_{n+1}^{*}=P_{n}^{*} A_{n}^{*}$, which implies that $P_{n+1}^{*}\left(A_{n}^{*}\right)^{-1}=\left(A_{n}^{*}\right)^{-1} P_{n}^{*}$.

Remark. The following theorem represents the extension of Theorem 3.1 for the case of the adjoint system $(\mathfrak{Y})$. This result is a natural extension of Proposition 6.1.1 from [3] for the case of LVT systems with GET. The solution is very similar to that used for Theorem 3.1, and is therefore omitted. This property can be easily checked also for Theorems 3.2 and 3.3.

Theorem 4.1. Assume that LTV system (') has a GET with supplementary projection sequences $\left(S_{m}^{k}\right)_{m \in \mathbb{Z}}, k \in\{1,2,3\}$, constants $D \geq 1, p \in(0,1)$ and strictly positive sequence $\left(a_{n}\right)_{n \in \mathbb{Z}}$, as in Theorem 3.1. Then the adjoint system (Y) also has a GET with supplementary projection sequences $\left(\left(S_{m}^{k}\right)^{*}\right)_{m \in \mathbb{Z}}$, $k \in\{1,2,3\}$, constants $D \geq 1, p \in(0,1)$ and strictly positive sequence $\left(a_{n}\right)_{n \in \mathbb{Z}}$ satisfying (1) and (2). More precisely, we have

$$
\begin{gathered}
\left\|V_{m} V_{n}^{-1}\left(S_{n}^{1}\right)^{*}\right\| \leq D p^{t_{m n}}, \quad n \geq m, \\
\left\|V_{m} V_{n}^{-1}\left(S_{n}^{2}\right)^{*}\right\| \leq D p^{t_{n m}}, \quad m \geq n, \\
\left\|V_{m} V_{n}^{-1}\left(S_{n}^{3}\right)^{*}\right\| \leq D p^{t_{n m}}, \quad 0 \geq m \geq n, \\
\left\|V_{m} V_{n}^{-1}\left(S_{n}^{3}\right)^{*}\right\| \leq D p^{t_{m n}}, \quad n \geq m \geq 0 .
\end{gathered}
$$

Acknowledgement (I.-L. Popa) This work was supported by the grants of the Romanian National Authority for Scientific Research and Innovation, CNCS/CCCDI - UEFISCDI, projects number PN-III-P2-2.1-PED-2016-1835 and PN-III-P1-1.1-MC-2017-2172, within PNCDI III. 
CHARACTERIZATIONS OF GENERALIZED EXPONENTIAL

\section{References}

[1] A.I. Alonso, J. Hong, R.Obaya, Exponential dichotomy and trichotomy for difference equations, Comput. Math. Appl. Vol. 38 (1999), 41-49.

[2] A. Castaneda, G. Robledo, A Topological Equivalence Result for a Family of Nonlinear Difference Systems Having Generalized Exponential Dichotomy, J. Difference Equ. Appl., DOI:10.1080/10236198.2016.1192161

[3] L. Dingjun, W. Xian, Z. Deming, H. Maoan, Bifurcation Theory and Methods od Dynamical Systems, Advenced Series in Dynamical Systems, Vol. 15, World Scientific, 1997

[4] A. Ducrot, P. Magal, O. Seydi, Persistence of Exponential Trichotomy for Linear Operators: A Lyapunov-Perron Approach, J. Dynam. Differential Equations, Vol. 28 (2016), 93-126.

[5] S. Elaydi, K. Janglajew, Dichotomy and Trichotomy of Difference Equations, J. Difference Equ. Appl. Vol. 3 (1998), 417-448.

[6] N. Lupa, M. Megan, Generalized exponential trichotomies for abstract evolution operators on the real line, J. Funct. Spaces Appl., Vol. 2013, Article ID 409049, 8 pages, 2013.

[7] C.L. Mihit, M. Megan, T. Ceausu, The Equivalence of Datko and Lyapunov Properties for $(\mathrm{h}, \mathrm{k})$-Trichotomic Linear Discrete-Time Systems, Discrete Dyn. Nat. Soc. Volume 2016, Article ID 3760262, 8 pages

[8] G. Papaschinopoulos, A Linearization result for a differential equation with piecewise constant argument, Analysis, 16 (1996), 161-170.

[9] G. Papaschinopoulos, On exponential trichotomy of linear difference equations Appl. Anal. 40 (1991), 89-109.

[10] I.-L. Popa, T. Ceausu, Generalized exponential trichotomy for difference equations, IEEE 11th International Symposium on Applied Computational Intelligence and Informatics, 2016, 173-176.

[11] I.-L. Popa, T. Ceauşu, O. Bagdasar, Characterizations of generalized exponential trichotomies for linear discrete-time systems, Electron. Notes Discrete Math., 56 (2016), 7-13.

[12] I.-L. Popa, M. Megan, T. Ceauşu, On h-Trichotomy of Linear DiscreteTime Systems in Banach Spaces, Acta Univ. Aplulensis Ser. Mat. Inf., 39 (2014), 329-339. 
[13] I.-L. Popa, T. Ceauşu, M. Megan, Characterizations of the $(h, k, \mu, \nu)$-Trichotomy for Linear Time-Varying Systems, Math. Methods Appl. Sci., 40 (17), 2016, 6172-6177.

[14] C. Vidal, C. Cuevas, $W$ ighted Exponential Trichotomy of Linear Differential Equations, Proceedings of the Conference on Differential \& Difference Equations and Applications, 2006, 1077-1086.

[15] Z.-G. Li, X.-Q. Song, X.-L. Yang, On nonuniform polynomial trichotomy of linear discrete-time systems in Banach spaces, J. Appl. Math., vol. 2014, Article ID 807265, 6 pages, 2014.

Ioan-Lucian Popa,

Department of Exact Science and Engineering,

"1 Decembrie 1918" University of Alba Iulia, 510009-Alba Iulia, Romania.

Email: lucian.popa@uab.ro

Traian Ceauşu,

Department of Mathematics, West University of Timişoara,

300223-Timişoara, Romania.

Email: ceausu@math.uvt.ro

Ovidiu Bagdasar,

School of Computing and Mathematics,

University of Derby,

Kedleston Road, Derby, DE22 1GB, UK.

Email: O.Bagdasar@derby.ac.uk

Ravi P. Agarwal,

Department of Mathematics,

Texas A\&M University-Kingsville,

700 University Blvd., MSC 172,

Kingsville, Texas 78363-8202.

Email: Ravi.Agarwal@tamuk.edu 